\title{
Coordinating Synchronous Interdependent Decisions in Complex Projects
}

\author{
Meriam Kilani $^{1}$, Franck Marle ${ }^{1}$, Ludovic Alexandre Vidal ${ }^{1}$ \\ ${ }^{1}$ Laboratoire Génie Industriel, CentraleSupélec, Université Paris-Saclay \\ 3 rue Joliot-Curie, 91170 Gif-sur-Yvette, France
}

\begin{abstract}
Oil and gas projects, due to their complexity, present a wide range of opportunities and issues that challenge decision-making processes. The major challenge addressed here concerns the coordination of multiple interdependent decisions. To overcome this challenge, we have explored two resolution strategies. The first one is a strategy by synchronous decision about a set of interdependent project decisions, the second one is by deciding one by one (asynchronous decisions). We present here the first strategy, including two similar modeling methods that can be conducted for such strategy: a QFD approach or a morphological approach. Both are followed by a multicriteria analysis. In the first stage, a set of scenarios is generated, then consistency between different decision alternatives is investigated using compatibility matrix. In the second stage, an evaluation and selection of an appropriate scenario is performed. Finally, to illustrate this strategy, an example is presented. This paper concludes that a structured methodology is promising for such contexts and draws some perspectives for asynchronous strategy and for the global strategy choice.
\end{abstract}

Keywords: oil and gas development projects, Exploration \& Production megaprojects, Decision Modeling, DSM, compatibility of decisions, Morphological analysis, Multi-criteria decision, $Q F D$

\section{Introduction}

Oil and gas projects are challenging for multiple reasons. They can be technically complex as they may be carried out in inaccessible or difficult areas and their design activities can be highly dependent of the reservoir location (Salama et al. 2008). They must respect strict budgets and demanding schedules that do not always match reality (Elhoush and Kulatunga 2017; Merrow and Analysis 2012). They have to deal with the geopolitical and economic vagaries of oil and gas. They are uncertain and risky given that geoscientific uncertainties are considerable and subsoil resources are only known at the end of the field's life. Another major challenge in such projects, is that development studies and data acquisition may require years to complete. However, during this period, certain decisions may be made without considering all the implications they may have, since their impacts cannot be easily anticipated at the time of decision making but may only become evident years later. As a result, at a certain point in the decision-making process, close to the Final Investment Decision (FID), it becomes surprising that many decisions have a huge impact, whether technical, security or financial. In addition, these projects involve multiple and diverse 
stakeholders, including the operating company, political organizations actors, contractors, subcontractors, suppliers, partners, host countries, and non-governmental organization actors (Eweje et al., 2012; VENTROUX, 2015). They finally have to deal with organizational problems since these projects requires the collaboration and coordination of actors from different domains, and coordination that crosses traditional organizational boundaries (Merrow and Analysis, 2012; Salama et al., 2008; VENTROUX, 2015).

Given the nature of oil and gas projects and all the issues mentioned above, all of this can lead to complexity in managing the actors, the interfaces between them and their relationships. The coordination of decisions taken during the project may also be difficult (Ventroux et al., 2017), especially the initial ones, since they structure what can be done in the future and condition the success or failure of the project. The coherence and compatibility of certain decisions, especially those that must be taken collectively, and the control of their impacts can also pose a major problem in the management of such projects.

Therefore, the objective of this paper is to propose decision-making strategies that are specific to interdependent decisions context. The aim of such strategies is to improve coordination between different decisions by ensuring consistency between their alternatives and improve communication between the different actors involved in these decisions. The remainder of the paper is as follows. Section 2 introduces the context and research questions. Section 3 presents the possible strategies to respond to those. Section 4 introduces related work on decision structure matrix, QFD approach, morphological analysis and multi-criteria approach. Section 5 details the first phase of the proposed process with an illustrative example. Section 6 details the second phase of the proposed process which includes possible decision-making strategies for selecting the best performing scenario. Finally, section 7 draws some conclusions and next steps.

\section{Context and Research Question}

In this section, we present the industry context and the issues related to the management of complex oil and gas development projects. We focus on the upstream phases of the project which includes basically the first three phases of development prior to the sanctioning of the project: the conceptual phase, the pre-project phase and the basic engineering phase. The main elements addressed in this paper are the decisions, their interdependences, and their alternatives.

In order to better comprehend the different challenges related to decision making in our context, we decided to elaborate a questionnaire intended for the main actors involved in the decision-making process (Project Managers, Architecture Manager, Procurement and Equipment Manager, Global Category Manager, Facilities and Engineering Manager etc.). This questionnaire was designed in a generic way, without specifying either the phase of development of the project or the entity to be questioned. The questionnaire is composed of 18 qualitative questions categorized into 6 categories: 1/ general questions; 2/ how interactions between decisions are managed; 3/ how impacts of decisions are managed; 4/ 
how the notion of decision temporality is managed; $5 /$ how the uncertainty related to a decision is managed; $6 /$ the risks resulting from already made decisions.

It emerged from these interviews that oil and gas development projects are multidimensional, and thus demand multi-stakeholder and collaborative efforts, with contributors from various backgrounds and continents. This leads to complex relationships between project stakeholders and highly independent decisions with multiple, potential links of different intensity and nature. In addition, a coherent set of decisions, prior to the sanctioning of the project, is important to the success of these projects, particularly given their potential impact that can propagate to the next phases of development. Based on precedent experiences, a consistent set of decisions can lead to multiple benefits and advantages. It allows to avoid blockages in the implementation phase, elaborate a development concept that is compatible with the requirements of the studied context and that respects the expectations of the host country. Therefore, to avoid inconsistencies and backtracking, which can be very costly for the project, we assume that it is necessary to have a global vision of decisions to be made. However, the questions that arise are: how to choose between the different alternatives for multiple decisions while ensuring their compatibility and performance? how to ensure a coherent set of decisions?

\section{Solving Strategies}

It seemed to us that two resolution strategies for making multiple decisions are possible in the first place. The first one is a packet-synchronous strategy as illustrated in Figure 7, that consists in focusing on a subset of interdependent decisions to coordinate them synchronously with its environment.

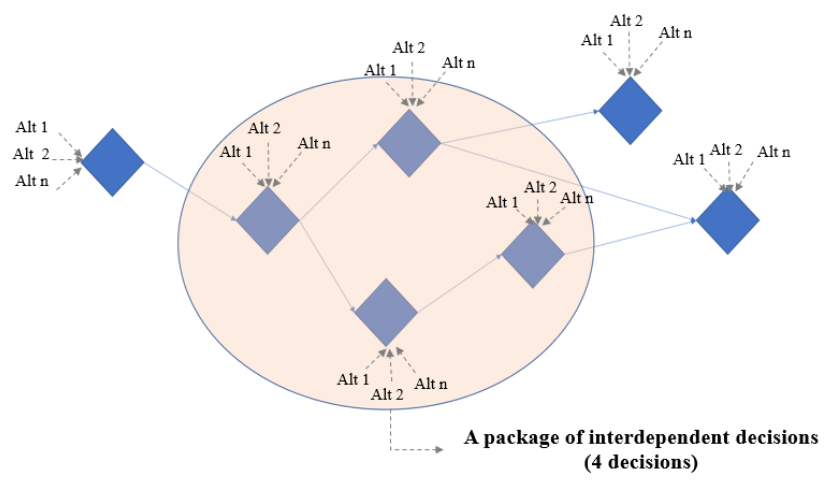

Figure 7: Synchronous strategy, focused on a package of interdependent decisions

The second one is an asynchronous strategy (Figure 8) that consists in focusing on a single decision while considering its coordination with its (at least) direct environment. It is important to point out here that these strategies are not intended to be universal, but the strategy of resolution may change depending on the studied context. 
The present article focuses on the first strategy which is detailed here with an illustrative example based on our industrial context. This strategy will improve communication and coordination in making synchronous interdependent decisions. The other strategy and the global assistance to strategy choice are developed in parallel but not included in this paper.

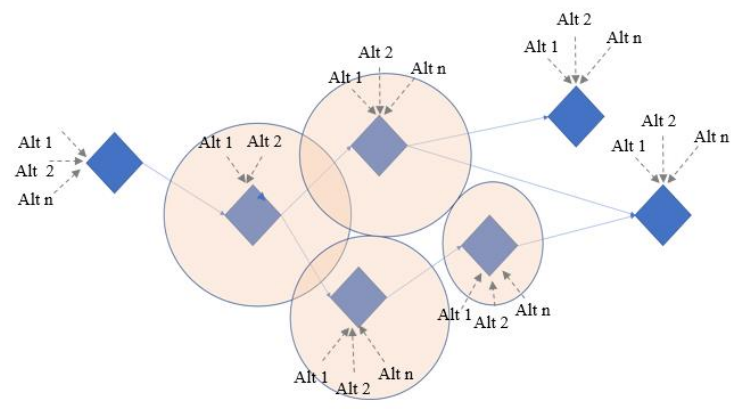

Figure 8: Asynchronous strategy, focused on a decision and its environment

In the field of multiple decisions, it has been established that dealing with graphs could be difficult, especially where a significant number of domains of decision making is involved (OMG, 2019). We thus use the well-known matrix-based approach DSM. Four matrices are modeled here:

- A Decisions x Decisions DSM for identifying possible interdependence between decisions (an illustration extracted from literature is given in Figure 3 Section 4.1).

- A Decisions x Decision Alternatives DMM for identifying and structuring possible alternatives for each decision (an example in our context is introduced in Figure 4 Section 5).

- A Decision Alternatives $\mathbf{x}$ Decision Alternatives DSM for identifying and assessing compatibility degree between each couple of decision alternatives (an example in our context is given in Figure 5 Section 5).

- A Decision Alternatives $x$ Project Objectives DMM for identifying performance of decision alternatives.

The final decision will be a scenario, a decision vector, where cells for each decision correspond to respectively selected alternative. The originality is of course to consider not only performance but also compatibility between decision alternatives to get a coherent and performing scenario. 


\section{Related Work}

\subsection{Decision structure matrix}

DSM (Dependency and Structure Modeling) is a technique that allows the objects of a project to be modeled, notably decisions in our context (Campagna et al., 2020). They proposed to model the global decision environment and not only the decisions themselves using DSM, as dealing with graphs could be difficult, especially where a significant domain of decision making is involved (OMG, 2019). The elements that are essential for decision making and the dependencies between them are modeled in this matrix. These elements include, a decision element, a Business Knowledge Model (BKM) element, an input data element, a knowledge source element, and a decision service element. The three types of requirements, presented in this matrix, and used to reflect the dependencies between these elements are defined as follows: an information requirement, a knowledge requirement, and an authority requirement. Each element of the matrix is modeled with a different color while the requirements are modeled with different symbols. For example, in the following matrix shown in Figure 9:DSM for modeling the decision environment ( Extract from (Campagna et al. 2020)), the decisions are labeled with a yellow color, the input data with a gray color. As for the requirements, the authority's requirements are marked by black circles, the information requirements by black triangles.
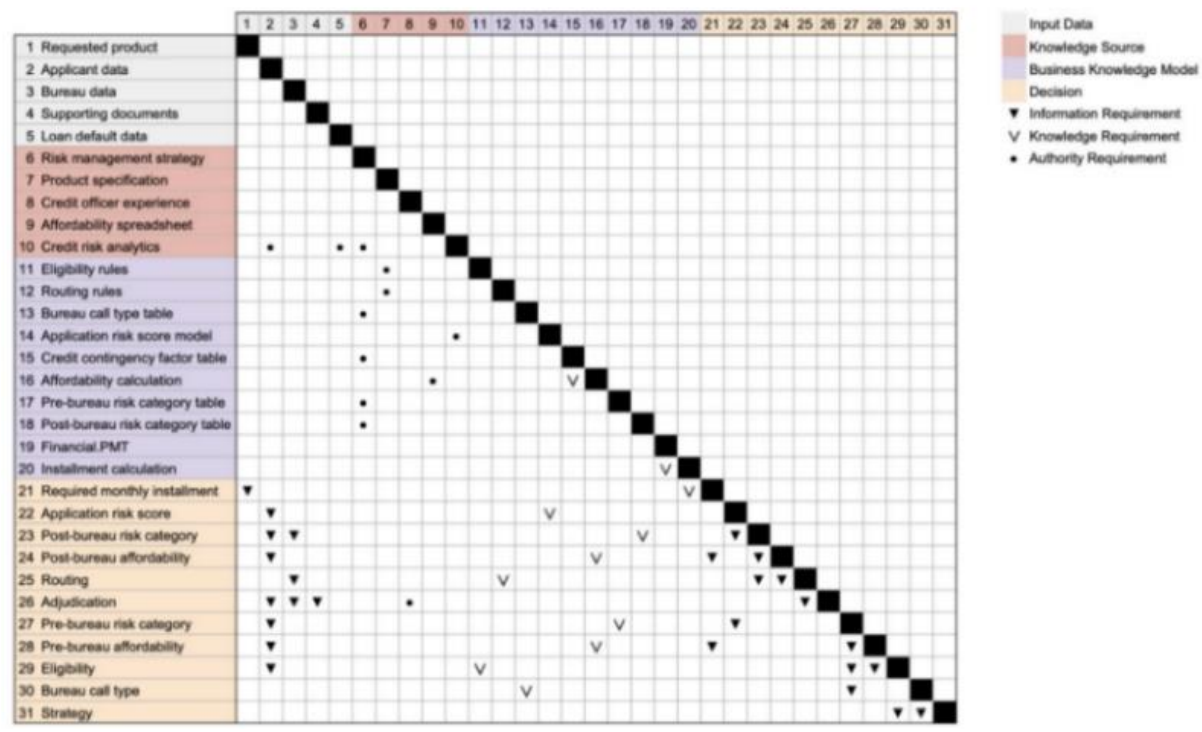

Figure 9:DSM for modeling the decision environment ( Extract from (Campagna et al. 2020)) 


\subsection{QFD approach}

The QFD (Quality Function Deployment) approach presents a possible alternative to be adopted in the packet-synchronous strategy. It presents a decision support tool for the design of products or services which provides a guide to effectively define customer requirements and convert them into detailed technical specifications to produce new products that meet those requirements (Bevilacqua et al., 2006). It has traditionally been widely used for decision-making (Van De Poel, 2007) and as a multifunctional design tool (Raissi et al., 2011) in product development and production (Prior and Akao, 1967). According to (Singgih et al., 2013). QFD can provide some short-term benefits such as reducing challenges associated with poor coordination between the different work departments and helping to change the corporate culture. Finally, this approach allows the construction of scenarios based on several interdependent alternatives and considering their performance as well as their compatibility.

\subsection{Morphological analysis and multi-criteria approach}

The second possible technique for synchronous decision-making is to consider compatibility, then performance, instead of considering them simultaneously as in QFD. The first phase is called morphological analysis and aims at identifying the different possible configurations using a morphological matrix (Im and Cho 2013). The second phase is a multi-criteria approach used to evaluate and select the best scenario in terms of performance among those obtained in the first phase.

\subsubsection{First phase: morphological analysis}

The morphological analysis or the general morphological approach (GMA) is a modeling method that aims to structure and explore the whole set of relationships or "configurations" involved in multidimensional and generally unquantifiable complex problems (Ritchey, 2006). This approach is a 2-phase process that involves analysis and synthesis. The analysis phase is subdivided into two steps. The first analysis step consists of identifying the most relevant criteria, also known as plausibility criteria, or parameters to describe the problem (Duczynski, 2004). The second step consists of identifying a spectrum of values or conditions for each criterion (Ritchey 2012, 2015). Once this identification is done, a morphological field can be constructed, and an n-dimensional configuration space can be created (Ritchey 2012, 2015). For this purpose, a matrix is constructed, where the different parameters describing the problem are placed in columns and their conditions in rows. A possible configuration or scenario is identified by selecting a single value for each parameter (Ritchey, 2006). The main objective of this step is to examine all the configurations.

The next phase aims to converge to a shortlist of most plausible scenarios or configurations through identifying conflicting or otherwise incompatible internal relationships between the levels of conditions. A process of Cross-Coherence Assessment (CCA) is performed, 
where all the relationships between the levels of parameters in the morphological matrix are examined and compared to each other, in pairs, like a cross-impact matrix, so that consistent and compatible configurations can be synthesized (Ritchey 2012, 2015). Several methods can be identified at this stage, among which the plausibility index method that will be detailed below (Duczynski, 2004; Hafezi et al, 2017). Consequently, conditions pairs that are not consistent will be eliminated (Im and Cho 2013). The objective of this step is to reduce the total set of possible configurations in the morphological matrix to a smaller, more manageable set of internally consistent combinations (Im and Cho, 2013). This leads to a set of compatible scenarios, which must be compared in terms of performance.

\subsubsection{Second phase: multi-criteria analysis to identify the best scenario}

The second phase in the process is to examine plausible scenarios in detail using multicriteria approaches to deduce the best ones (Im and Cho 2013; Montibeller et al., 2006). This phase is also subdivided into three sub-phases. The first sub-step consists of identifying the criteria that will be used to evaluate the most plausible scenarios identified in the previous phase. It should be noted here that these criteria are different from those mentioned in the morphological analysis of the first phase. These criteria are rather performance and efficiency criteria that are not related to the description of the scenarios. The second sub-step consists in identifying the adequate multi-criteria method to be used to evaluate the performance of the different scenarios. The third sub-step aims to describe the process that will lead to the convergence to the best scenario in terms of robustness and efficiency.

\section{Problem Modeling}

In the previous section, we presented the general model we intend to adopt for solving our problem, based on modeling of interdependence between decision alternatives (for compatibility assessment), and between decision alternatives and project objectives (for performance assessment). QFD and morphological analysis are two possible methods that have similar aspects, their main difference being the simultaneity or sequence of consideration of both parameters. In this section, an example of application of morphological method is provided, however in reality both methods are considered by decision-makers. In our case, the criteria of the morphological analysis identified in the first step will model six decisions to be made (Figure 10), designated here by $D i, i=1$ to 6 . For the example illustrated here, the first column of the morphological matrix corresponds to a oil and gas development concept decision where 5 alternatives are proposed. Two types of concepts are mainly presented in this illustration: fixed concepts and mobile concepts. Second and third columns are about wells (architecture and number) with 4 alternatives for each. The fourth column represents a contracting strategy decision where four alternatives are proposed. For instance, the fourth alternative consists of assigning basic engineering, detailed engineering, procurement and construction management to a first contractor, and the design of the construction phase to a second contractor. The fifth column corresponds 
to a local content decision that is related to the set of local resources that a company must use or develop along its value chain when investing in a project in a host country.

\begin{tabular}{|c|c|c|c|c|c|}
\hline Development concept & \begin{tabular}{|c|} 
Well \\
architecture \\
\end{tabular} & \begin{tabular}{|l} 
Number \\
of wells
\end{tabular} & Contractual strategy & Local content strategies (LC) & Strategies In-Country-Value (ICV) \\
\hline $\begin{array}{l}\text { Fixed platform: Jacket- } \\
\text { deck }\end{array}$ & Vertical & 4 & $\begin{array}{l}\text { (OBT) EPC Lump Sump with (CFT) } \\
\text { C not fixed Reimbursable }\end{array}$ & $\begin{array}{c}\text { Action of production adapted } \\
\text { to the local offer }+ \text { action of } \\
\text { improvement of the human } \\
\text { capital (maximum) }\end{array}$ & Ensure access to water \\
\hline $\begin{array}{l}\text { Fixed platform: } \\
\text { Gravitary platform }\end{array}$ & Directional & 6 & $\begin{array}{c}\text { (CFT)E Reimbursable +(CFT)P } \\
\text { Reimbursable + (CFT)C C I\&I Com } \\
\text { Reimbursable }\end{array}$ & $\begin{array}{l}\text { Action of production lower } \\
\text { than the local offer + action of } \\
\text { improvement of the human } \\
\text { capital (minimal) + Actions } \\
\text { related to the local industries }\end{array}$ & $\begin{array}{l}\text { Power generation (creation of a } \\
\text { domestic gas sector) }\end{array}$ \\
\hline $\begin{array}{l}\text { Fixed platform:Tension } \\
\text { Leg Plateforms (TLP) }\end{array}$ & Horizontal & 8 & $\begin{array}{l}\text { (CFT) EPCm Lump Sump +C } \\
\text { Reimbursable }\end{array}$ & $\begin{array}{l}\text { Action of production superior } \\
\text { to the local offer + Action of } \\
\text { local employment (maximum) }\end{array}$ & $\begin{array}{l}\text { Construction of transport } \\
\text { infrastructures }\end{array}$ \\
\hline $\begin{array}{l}\text { Mobile platforms and } \\
\text { floating units: FPSO }\end{array}$ & Multilateral & 10 & $\begin{array}{c}\text { (CFT) EP lump Sump +(CFT) C T\&I } \\
\text { Com Reimbursable }\end{array}$ & & Creation of schools \\
\hline $\begin{array}{l}\text { Mobile platforms and } \\
\text { floating units:Semi- } \\
\text { submersible }\end{array}$ & & & & & \\
\hline
\end{tabular}

Figure 10: A morphological space with 6 parameters (decisions). The shaded cells represent one configuration among the 3840 possible configurations.

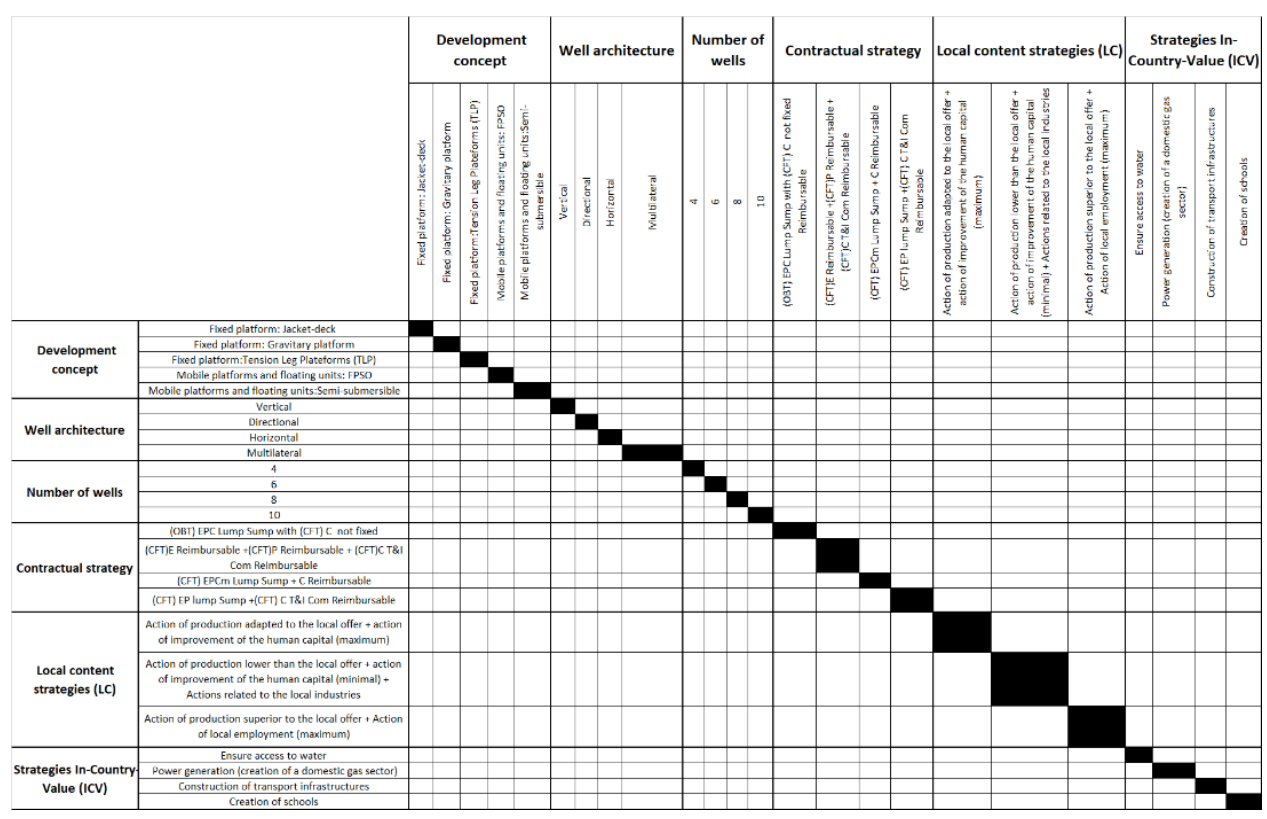

Figure 11: Example: Cross-consistency matrix for the morphological field in figure 4

After completing the two analysis sub-steps, the focus shifts to the third sub-step, which is a synthesis step aimed at converging on a short list of the most plausible scenarios or configurations. The assessment of whether, or to what extent, the pair of alternatives (conditions) can represent a consistent relationship is carried out for each pair of 
alternatives under consideration (Ritchey 2012, 2015). For this evaluation, we use plausibility indices to evaluate pairs of alternatives. For each possible intersection in terms of consistency, a plausibility index is proposed in the corresponding cell and similarly for those that are inconsistent. In the following compatibility matrix, we have adapted the three plausibility indices proposed by (Im and Cho, 2013), but we have just modified the "-" index (hyphen) by the letter "P" which marks a good match between the pairs, or that the pair is optimal. The other indices are maintained as they are: " $\mathrm{K}$ " to designate that the pairs are possible, and they could match, but not in an optimal way; " $\mathrm{X}$ " to indicate that the pairs are impossible or that it is a very bad idea. The two blocks shaded in blue correspond to decisions that are independent. It is important to note here that the main objective is to illustrate what a compatibility matrix looks like. The way in which the matrices on the diagonal are filled in mainly depends on the nature of the decision, and whether it is acceptable or not to choose more than one alternative per decision. Indeed, some decisions are exclusive, which means that only one alternative must be chosen per decision. For example, for the decision concerning the number of wells, only one alternative can be chosen, either $4,6,8$ or 10 wells. It is the same for the decision concerning the development concept. Therefore, in the following figure, the matrices corresponding to these decisions are only filled with " $\mathrm{X}$ ", which means that the interactions between the alternatives contained in these matrices cannot be examined. However, for some decisions, a combination or mix of alternatives is possible. For example, for the decision related to the In-Country-Value strategies, it is possible to combine two alternatives: one action to provide access to water and another to create schools. For the matrices corresponding to these decisions, the compatibility between the different alternatives can be studied to determine whether they are compatible with each other or not. Once these indices are assigned, as shown in Figure 11, consistent configurations can be identified and then evaluated at a later stage.

\section{Coordination Strategies}

So far, a set of plausible scenarios was identified either through morphological analysis or the QFD approach. However, it remains unclear which of these scenarios should be retained in terms of performance. This section serves to detail the decision-making strategies employed to select the best-performing scenario and to illustrate how to coordinate in making that decision. In our model, this step corresponds to the second phase which is a multi-criteria analysis that aims at identifying the best scenario in terms of performance. Several multicriteria decision methodologies exist in the literature that allow choices to be deduced from data resulting from another phase.

For each decision owner, the inputs are information about other decisions within and outside the group (upstream and downstream), since there is a strong coordination with decisions within the group, however there is also a light coordination expected with decisions outside the group, either directly or indirectly connected. Different tools and techniques exist for convergence, like collaborative decision-making, scenario generation and evaluation, distributed authority-based techniques. Finally, the output of this process is a vector of decisions, with a decision alternative for each decision, and possibility a degree of firmness associated to a degree of confidence in the proposed decisions. The 
Meriam Kilani, Franck Marle, Ludovic Alexandre Vidal

firmness gives an indication to future decisions about the possibility of proposing changes in the current scenario.

\section{Conclusion}

In this paper, we explored a new strategy for making multiple decisions based on a compatibility study between decision alternatives using DSMs. We showed how one can create a morphological matrix and a compatibility matrix and use them for building compatible and performing multi-decision scenarios. This approach, according to (Ritchey 2015), can offer numerous advantages. First, it seeks to objectively reduce the number of solutions for a given problem. Second, it can support and facilitate the identification of further relationships or scenarios that may not be immediately evident. Moreover, this approach can bring improvements in terms of communication and coordination between the different entities involved in the project.

We have mainly presented the synchronous packet resolution strategy which aims to focus on a subset of interdependent decisions in order to coordinate them synchronously with its environment. What we plan to do in the next steps is to test this strategy on a detailed case study to validate it, then study in detail the asynchronous approach. However, it seemed relevant also to propose a third strategy which consists in superposing these two strategies on the same decision network. This strategy was decided to be called a hybrid strategy. Many questions arise at this stage, first how to choose an appropriate strategy and how to know which strategy will best suit my context, since, as mentioned at the beginning, each decision strategy is adapted to a well-defined context, and finally, are there any selection criteria that can guide our choice?

\section{References}

Bevilacqua, M., F. E. Ciarapica, and G. Giacchetta. 2006. "A Fuzzy-QFD Approach to Supplier Selection." Journal of Purchasing and Supply Management 12(1): 14-27.

Campagna, Dario et al. 2020. "Using DSMs for the Visualization and Analysis of Decision Models in Business Processes." (1): 10-10.

Duczynski, Guy. 2004. "Systems Approaches to Economic Development for Indigenous People: A Case Study of the Noongar Aboriginals of Australia." Futures 36(8): 869-88.

Elhoush, R., and U. Kulatunga. 2017. "The Effectiveness of Project Risk Management: A Study within the Libyan Oil and Gas Industry." University of Salford Institutional Repository: 68091.

Eweje, John, Rodney Turner, and Ralf Müller. 2012. "Maximizing Strategic Value from Megaprojects: The Influence of Information-Feed on Decision-Making by the Project Manager." International Journal of Project Management 30(6): 639-51. http://dx.doi.org/10.1016/j.ijproman.2012.01.004.

Hafezi, Reza, Amir Naser Akhavan, and Saeed Pakseresht. 2017. "Projecting Plausible Futures for Iranian Oil and Gas Industries: Analyzing of Historical Strategies." Journal of Natural Gas Science and Engineering 39: 15-27. http://dx.doi.org/10.1016/j.jngse.2016.12.028.

Im, Kwanyoung, and Hyunbo Cho. 2013. "A Systematic Approach for Developing a New Business Model Using Morphological Analysis and Integrated Fuzzy Approach.” Expert Systems with Applications 40(11): 4463-77. http://dx.doi.org/10.1016/j.eswa.2013.01.042. 
Merrow, Edward W, and Independent Project Analysis. 2012. "De Meyer2002 - A Framework for Project Management under Uncertainty." (April): 38-42.

Montibeller, Gilberto, Haidee Gummer, and Daniele Tumidei. 2006. "Combining Scenario Planning and Multi-Criteria Decision Analysis in Practice Journal of Multi-Criteria Decision Analysis Volume 14, Issue 1-3." Journal of Multi-Criteria Decision Analysis 14(1-3): 5-20. http://onlinelibrary.wiley.com/doi/10.1002/mcda.403/abstract.

OMG. 2019. "Decision Model and Notation (DMN ) Specification." 0: 1-171.

Van De Poel, Ibo. 2007. "Methodological Problems in QFD and Directions for Future Development." Research in Engineering Design 18(1): 21-36.

Prior, AN, and Y Akao. 1967. "QFD: Past, Present and Future." (2): 1-12. http://esslli2013.de/wpcontent/uploads/2013/08/blackburn.pdf.

Raissi, S., M. Izadi, and S. Saati. 2011. "A Novel Method on Customer Requirements Preferences Based on Common Set of Weight." Australian Journal of Basic and Applied Sciences 5(6): $1544-52$.

Ritchey, Tom. 2006. "Problem Structuring Using Computer-Aided Morphological Analysis."

- 2012. "On the Formal Properties of Morphological Models *." 1(2): 1-16.

- 2015. "Applications of General Morphological Analysis." 4(1): 1-40.

Salama, Mohamed, Moustafa Abd, El Hamid, and Bill Keogh. 2008. "INVESTIGATING THE CAUSES OF DELAY WITHIN OIL AND GAS PROJECTS IN THE U . A . E ." (September): 819-27.

Singgih, Moses L, Dyah L Trenggonowati, and Putu D Karningsih. 2013. "Four Phases Quality Function Deployment ( Qfd ) By Considering Kano Concept , Time And.” International Conference on Engineering and Technology Development (2): 22-38.

VENTROUX, Julien. 2015. "Aide à La Maîtrise Des Risques Liés à La Contractualisation et l'exécution d'un Projet Complexe Pétrolier." : 1-198.

Ventroux, Julien, Ludovic Alexandre Vida, and Franck Marle. 2017. "Optimize the Supervision of Complex Projects by Taking into Account Interactions between Actors." Understand, Innovate, and Manage your Complex System! - Proceedings of the 19th International DSM Conference: 205-14.

Contact: F. Marle, Laboratoire Génie Industriel, CentraleSupélec, Université Paris- Saclay, 3 rue Joliot-Curie, 91170 Gif-sur-Yvette, FRANCE, franck.marle@ centralesupelec.fr 